\title{
Combined colorimetric and thermal analyses of reversible thermochromic composites using crystal violet lactone as a colour former
}

\author{
Ondrej Panák ${ }^{1}$, Markéta Držková ${ }^{*}$, Roman Svoboda ${ }^{2}$, Marta Klanjšek Gunde \\ 1 University of Pardubice, Faculty of Chemical Technology, Department of Graphic Arts and Photophysics, Studentská \\ 95, 53210 Pardubice, Czech Republic \\ 2 University of Pardubice, Faculty of Chemical Technology, Department of Physical Chemistry, Studentská 95,53210 \\ Pardubice, Czech Republic \\ 3 National Institute of Chemistry, Hajdrihova 19, SI-1001 Ljubljana, Slovenia \\ * Corresponding author: \\ Markéta Držková, E-mail: marketa.drzkova@upce.cz, Tel.: +420 466038 035, Fax: +420 466038031
}

\begin{abstract}
In present study, the intention was to investigate how the colour change of thermochromic composites is connected with their phase transitions. Crystal violet lactone was used as a colour former and bisphenol $\mathrm{A}$ as a developer. Two co-solvents, 1-tetradecanol and 1-tetradecanoic acid, were selected due to the same length of the aliphatic chain and different functionality. Series of seven thermochromic systems varying in co-solvent's molar ratio were prepared for each type of the cosolvent. The colour change was measured by reflectance spectroscopy and temperatures characterizing the dynamic colour change were computed from the cumulative colour difference function. The onset and endset temperatures of thermochromic system's phase transitions were obtained from DSC analyses. Connections between the fastest colour changes and the decolouration limits at raised temperatures to solid-solid, solid-liquid and liquid-solid transitions are demonstrated for both heating and cooling, respectively. Different behaviour of compared systems with alcoholic and acidic co-solvent is discussed as well.
\end{abstract}

\section{Keywords}

Reversible thermochromism, Crystal violet lactone, Cumulative colour difference, DSC, 1Tetradecanol, 1-Tetradecanoic acid

\section{Acknowledgement}

The work was supported by the Ministry of education, youth and sports of the Czech Republic, project LD14098 within COST CZ program.

\section{Introduction}

Three component leuco dye based thermochromic systems usually exhibit reversible colour change, which is dependent on their component ratios [1-3]. The colour change is driven by interaction of a colour former and a developer in an environment formed by a co-solvent and is related to the change in crystalline structure of the co-solvent molecules. The phase change of the co-solvent then determines the temperature at which the colour change appears [4-7]. Mostly long aliphatic chain 1alcohols were used in research of thermochromic systems; in our previous research [1-3], 1-alcohols with even number of carbon atoms were used. 
At low temperatures, 1 -alcohols have a crystalline structure that can be $\beta$-form or $\gamma$-form [8]. In $\beta$ form the molecules are nearly normal to the base of the unit cell and the cell is formed of the same number of trans- and gauche- molecules, while in the $\gamma$-form, the molecular chains are inclined about $60^{\circ}$ to the cell base and the cell is composed of trans- molecules only [8]. The oxygen atom and skeletal carbon atoms are coplanar in trans- molecule, whereas in gauche molecule the oxygen atom is out of the skeletal plane [8]. Both crystalline phases transform to a solid $\alpha$-phase [8] called also rotator phase [9-12]. In the rotator phase the molecules are layered nearly normal to the cell base [10]. The rotator phase is distinguished from the low temperature crystalline structure by lack of long-range order in the rotational degree of freedom of molecules about their long axis $[9,13]$. The crystalline $\beta$-form is predominant for 1 -alcohols with odd number of carbon atoms, and crystalline $\gamma$ form is predominant for 1-alcohols with even number of carbon atoms [8-10]; the infrared spectroscopy also suggests that alcohols with shorter alkyl chain (e.g. 1-tetradecanol) have predominant $\beta$-form [8]. The solid-solid (rotator-crystal) phase transition exhibits a strong hysteresis comparing to solid-liquid (rotator-liquid) phase transition [9, 11]. On heating, the peaks characterizing the solid-solid and solid-liquid transitions of 1-alcohols with even number of carbon atoms are overlapped in DSC data, whereas on cooling these transitions are clearly separated [10, 14].

Thang et al. [4] attributed the colouration of systems to formation of disordered rotator phase of the alcoholic co-solvent. Hajzeri et al. [3] supposed that the colour change on cooling is connected with transition from the rotator phase to a rigid $\gamma$-phase of the alcoholic co-solvent. On heating, the temperature at which the system becomes colourless was lower than the melting point of the system [3].

Burkinshaw et al. [6] investigated thermochromic systems incorporating fatty acids as the co-solvent. The colour change is reported as sharp, however without deeper temperature dependent colour analyses. The sharp colour change might be attributed to a single phase transition of fatty acids [1517]. Recently we have shown that the cumulative colour difference, representing the length of the path of colour change in CIELAB space, is a suitable parameter in an evaluation of colour change as a function of temperature [2]. Present study aims to investigate the connection between the temperature dependent dynamic colour change of thermochromic composites with their phase transitions. Two co-solvents matching in the length of aliphatic chain were chosen: 1-tetradecanol and 1-tetradecanoic acid. The main question relies temperatures characterising the colour change with temperatures characterising the phase change of the system.

\section{Experimental}

\section{Materials and sample preparation}

In formulation of ternary thermochromic composites, crystal violet lactone (CVL, 6(dimethylamino)-3,3-bis[p-(dimethylamino) phenyl] phthalide, $>95 \%$ ) was employed as a colour former and Bisphenol A (BPA, 2,2-bis(4-hydroxyphenyl)propane, > $99 \%$ ) was used as a developer. Two long-chain saturated fatty compounds with carbon number 14 were compared as a co-solvent, 1tetradecanol (TD, > $98 \%$ ) and 1-tetradecanoic acid (myristic acid, MA, >99\%), respectively. All chemicals were purchased from Tokyo Chemical Industry (Japan) and were used without further purification.

In case of each co-solvent, seven thermochromic systems were prepared as described in [2], varying in molar ratio of their components: $1 / 4 / 60,1 / 4 / 80,1 / 4 / 100,1 / 4 / 120,1 / 4 / 200,1 / 4 / 400$, and 1/4/600 $\left(x_{\mathrm{CVL}} / x_{\mathrm{BPA}} / x_{\mathrm{TD}}\right.$ or $x_{\mathrm{CVL}} / x_{\mathrm{BPA}} / x_{\mathrm{MA}}$, respectively). This range of concentrations has been chosen based 
on previous results [1-3]. Similarly, keeping the continuity with our previously published research was the reason to employ BPA as the developer, although it is widely discussed with respect to health risks in recent years, as summarized in [18].

\section{Characterization}

Phase transitions of pure co-solvent and thermochromic mixtures with varying molar ratio of their components, in total 16 samples in powder form, were characterized by TA Instruments DSC Q2000 instrument. All samples were examined in portions of approximately $5.5 \mathrm{mg}$ enclosed hermetically in low-mass Al pans. The rate of temperature change during DSC measurements was $2{ }^{\circ} \mathrm{C} \mathrm{min}{ }^{-1}$. The temperature interval ranged between 0 and $50{ }^{\circ} \mathrm{C}$ in case of samples with TD co-solvent and between 20 and $75^{\circ} \mathrm{C}$ in case of samples with MA co-solvent. Each sample was thermally treated in two heating/cooling cycles and the sample was tempered isothermally for 5 minutes at the end of each temperature ramp. Only the second cycle was considered in further computations. The temperature was determined as the temperature of the reference due to the limiting heat transfer of the sensor. Onset and endset temperatures of the main DSC peaks were determined as a cross-section of the linear extrapolations from the baseline and corresponding inflection points. These temperatures are in further marked e.g. as $T_{\mathrm{OH} 1}$ for onset temperature of the first DSC peak in a heating run and $T_{\mathrm{EC} 2}$ for endset temperature of the second DSC peak in a cooling run.

The colour analysis was done by measuring the bulk thermochromic composites using X-Rite EyeOne Pro spectrophotometer. The reflectance measurements were taken continuously with a $1{ }^{\circ} \mathrm{C}$ resolution in the temperature interval of $10-50^{\circ} \mathrm{C}$ in case of samples with $\mathrm{TD}$ co-solvent and 30 $70{ }^{\circ} \mathrm{C}$ in case of samples with MA co-solvent. The rate of temperature change was $1.5-2{ }^{\circ} \mathrm{C} \min ^{-1}$, i.e. close to the rate used during DSC measurements. For further details of experimental setup and determination of basic colorimetric parameters please refer to [2]. For all samples, temperature dependence of the cumulative colour difference $\Delta E_{\mathrm{C}}$ was calculated, representing dynamic colour change during heating as well as cooling [2]. Based on $\Delta E_{\mathrm{C}}$, six characteristic temperatures were determined (Fig. 1): the temperature at which the decolouration starts $\left(T_{\mathrm{DS}}\right)$ and ends $\left(T_{\mathrm{DE}}\right)$ on heating, together with the temperature of the fastest decolouration $\left(T_{\mathrm{Dmax}}\right)$; analogously, the temperature at which the colouration starts $\left(T_{\mathrm{CS}}\right)$ and ends $\left(T_{\mathrm{CE}}\right)$ together with the temperature of the fastest colouration $\left(T_{\mathrm{Cmax}}\right)$ on cooling. In finding these temperatures, gradients $G$ of $\Delta E_{\mathrm{C}}$ were used, computed according Equation (1). The temperatures of the fastest colour change correspond to the maximum of given $G(T)$. The temperatures of the start and stop of colour change were taken as the last temperature at which given $G(T)$ is below the threshold in low and high temperature range, respectively. The threshold was based on first (or last) 10 values of given $G(T)$ in corresponding range and set within 2 standard deviations from their average, as illustrated in Fig. $1 \mathrm{~b}$.

$G(T)=0.5\left|\Delta E_{\mathrm{C}}(T+1)-\Delta E_{\mathrm{C}}(T-1)\right|$ 

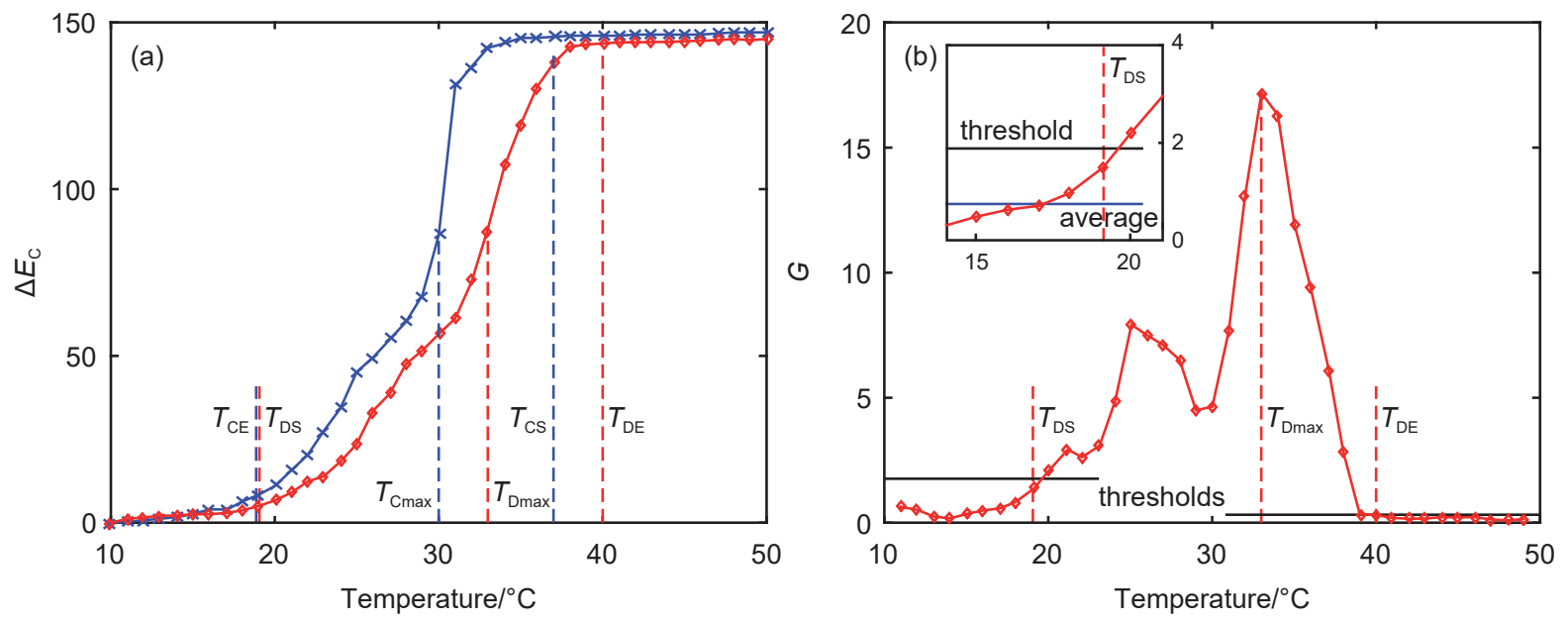

Fig. 1 Example of cumulative colour difference $\Delta E_{\mathrm{C}}$ temperature dependences for the thermochromic composite with $\mathrm{TD}$ as a co-solvent $\left(x_{\mathrm{CVL}} / x_{\mathrm{BPA}} / x_{\mathrm{TD}}=1 / 4 / 60\right)$ with marked characteristic temperatures $T_{\mathrm{DS}}, T_{\mathrm{Dmax}}, T_{\mathrm{DE}}, T_{\mathrm{CS}}, T_{\mathrm{Cmax}}$ and $T_{\mathrm{CE}}(\mathrm{a})$ and illustration of finding these temperatures for heating run from corresponding gradient $G(\mathrm{~b})$, where diamonds represent heating and crosses cooling of the sample

\section{Results and discussion}

\section{Thermal analysis}

The results of DSC measurement for samples containing TD and MA are presented in Fig. 2 and 3, respectively. Determined onset and endset temperatures of the main DCS peaks are listed in Table 1 for samples with TD and in Table 2 for samples with MA.
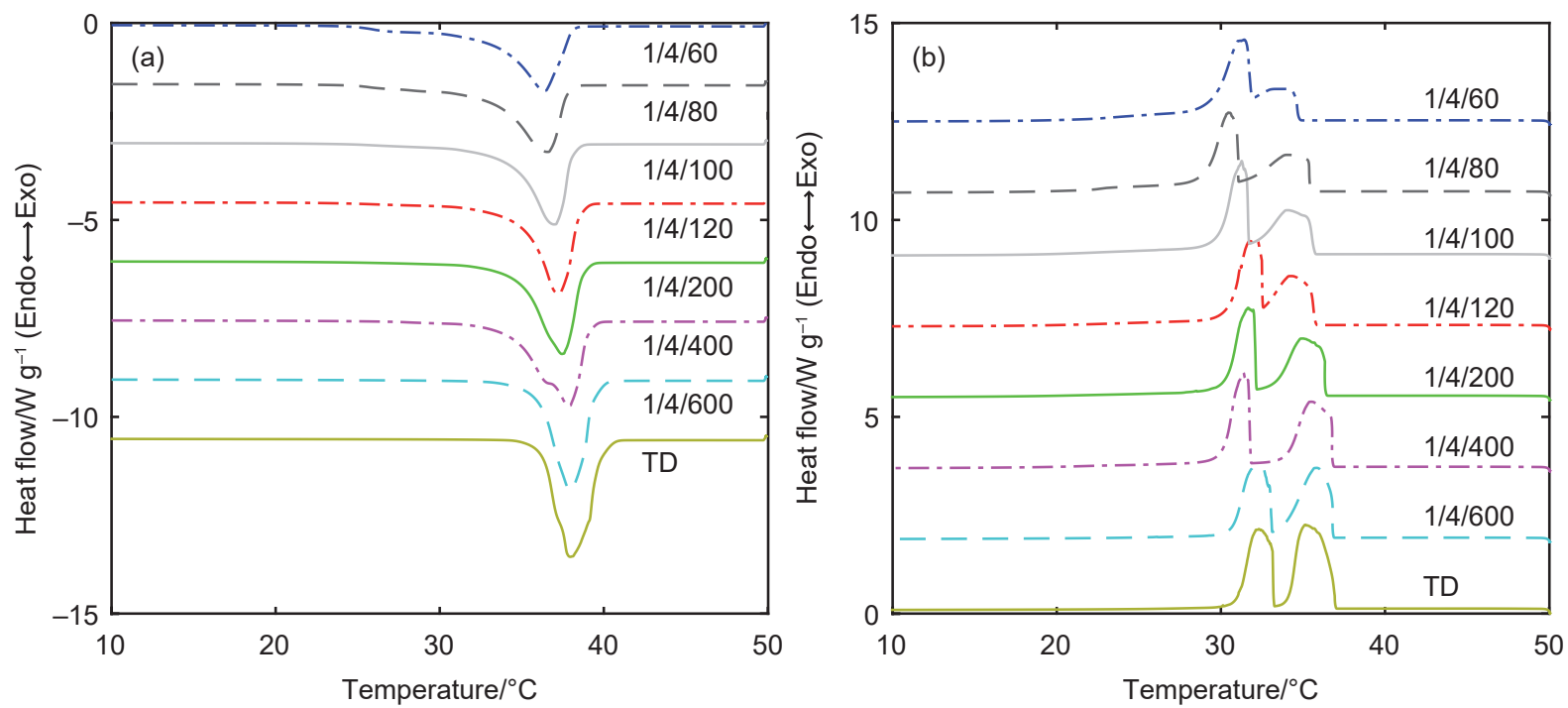

Fig. 2 DSC curves of the thermochromic composites with TD as a co-solvent and given $x_{\mathrm{CVL}} / x_{\mathrm{BPA}} / x_{\mathrm{TD}}$ and of the neat TD for heating (a) and cooling (b) run; the data are vertically displaced for clarity 

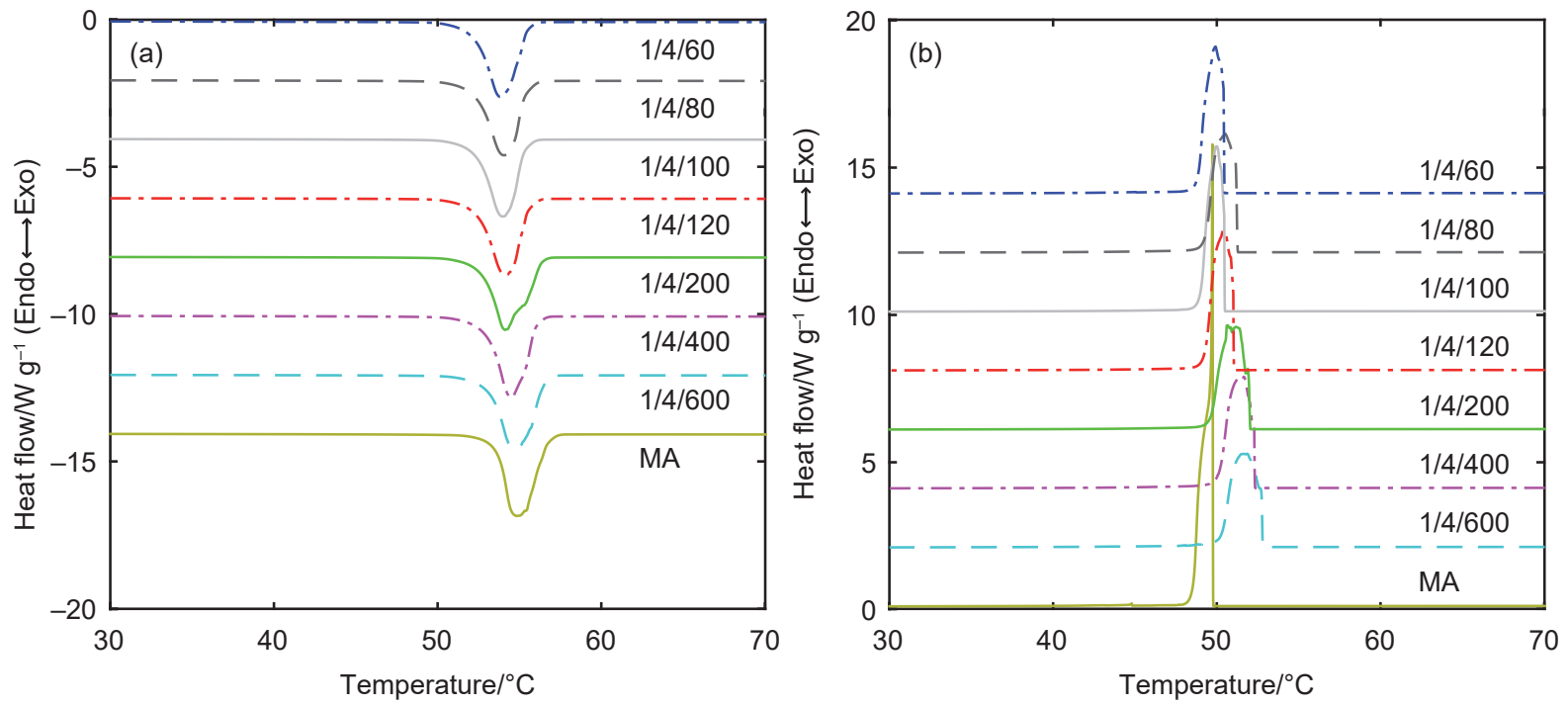

Fig. 3 DSC curves of the thermochromic composites with MA as a co-solvent and given $x_{\mathrm{CVL}} / x_{\mathrm{BPA}} / x_{\mathrm{MA}}$ and of the neat MA for heating (a) and cooling (b) run; the data are vertically displaced for clarity

Table 1 DSC results of neat TD and thermochromic composite powders with TD as a co-solvent determined onset $(\mathrm{O})$ and endset $(\mathrm{E})$ temperatures on heating $(\mathrm{H})$ and cooling $(\mathrm{C})$

\begin{tabular}{|c|c|c|c|c|c|c|c|c|}
\hline $\begin{array}{l}\text { Sample } \\
\left(x_{\mathrm{CVL}} / x_{\mathrm{BPA}} / x_{\mathrm{TD}}\right)\end{array}$ & $\begin{array}{l}\text { Endothermic } \\
\text { peak } 1 \\
T_{\mathrm{OH} 1} /{ }^{\circ} \mathrm{C}\end{array}$ & $\begin{array}{l}\text { Endothe } \\
\text { peak } 2 \\
T_{\mathrm{OH} 2} /{ }^{\circ} \mathrm{C}\end{array}$ & $\begin{array}{l}\operatorname{mic} \\
T_{\mathrm{EH} 2} /{ }^{\circ} \mathrm{C}\end{array}$ & $\begin{array}{l}\text { Exothern } \\
1 \\
T_{\mathrm{OC} 1} /{ }^{\circ} \mathrm{C}\end{array}$ & $\begin{array}{l}\text { nic peak } \\
T_{\mathrm{ECl} 1} /{ }^{\circ} \mathrm{C}\end{array}$ & $\begin{array}{l}\text { Exother1 } \\
2 \\
T_{\mathrm{OC} 2} /{ }^{\circ} \mathrm{C}\end{array}$ & $\begin{array}{l}\text { nic peak } \\
T_{\mathrm{EC} 2} /{ }^{\circ} \mathrm{C}\end{array}$ & $\begin{array}{l}\text { Exothermic } \\
\text { peak } 3 \\
T_{\mathrm{EC} 3} /{ }^{\circ} \mathrm{C}\end{array}$ \\
\hline $1 / 4 / 60$ & 24.8 & 33.3 & 38.1 & 34.6 & 29.7 & 31.9 & 29.3 & 20.6 \\
\hline $1 / 4 / 80$ & 24.8 & 33.2 & 37.8 & 30.7 & 31.0 & 31.0 & 28.9 & 21.1 \\
\hline $1 / 4 / 100$ & 24.5 & 34.4 & 38.1 & 35.6 & 31.8 & 31.7 & 29.8 & 19.1 \\
\hline $1 / 4 / 120$ & 24.2 & 35.2 & 38.5 & 35.7 & 31.9 & 32.5 & 30.4 & 18.7 \\
\hline $1 / 4 / 200$ & - & 34.8 & 38.7 & 36.3 & 33.1 & 32.1 & 30.2 & - \\
\hline $1 / 4 / 400$ & 26.7 & 34.2 & 39.1 & 36.7 & 33.9 & 31.8 & 30.1 & 18.2 \\
\hline $1 / 4 / 600$ & - & 36.0 & 39.5 & 36.7 & 33.6 & 33.1 & 30.6 & 23.2 \\
\hline Neat TD & - & 36.3 & 39.8 & 36.9 & 34.0 & 33.2 & 31.0 & - \\
\hline
\end{tabular}

Table 2 DSC results of neat MA and thermochromic composite powders with MA as a co-solvent determined onset $(\mathrm{O})$ and endset $(\mathrm{E})$ temperatures on heating $(\mathrm{H})$ and cooling $(\mathrm{C})$

\begin{tabular}{lllll}
\hline $\begin{array}{l}\text { Sample } \\
\left(x_{\mathrm{CVL}} / x_{\mathrm{BPA}} / x_{\mathrm{MA}}\right)\end{array}$ & $\begin{array}{l}\text { Endothermic peak } 1 \\
T_{\mathrm{OH} 1} /{ }^{\circ} \mathrm{C}\end{array}$ & $T_{\mathrm{EH} 1} /{ }^{\circ} \mathrm{C}$ & \multicolumn{2}{l}{ Exothermic peak 1} \\
\\
\hline $1 / 4 / 60$ & 52.2 & 55.6 & 50.5 & $T_{\mathrm{OC} 1} /{ }^{\circ} \mathrm{C}$ \\
$1 / 4 / 80$ & 52.3 & 55.4 & 51.3 & 48.8 \\
$1 / 4 / 100$ & 52.4 & 55.4 & 50.5 & 49.3 \\
$1 / 4 / 120$ & 52.4 & 55.6 & 51.1 & 49.0 \\
$1 / 4 / 200$ & 52.7 & 56.4 & 52.0 & 49.2 \\
$1 / 4 / 400$ & 53.0 & 56.1 & 52.3 & 49.6 \\
$1 / 4 / 600$ & 53.1 & 56.5 & 52.8 & 50.1 \\
Neat MA & 53.6 & 56.7 & 49.8 & 50.3 \\
\hline
\end{tabular}


It is apparent that the thermo-kinetic manifestation of the observed processes is significantly different for the two used co-solvents. Looking first at the DSC data obtained for TD at cooling, it is clear that the liquid $\rightarrow$ solid and solid $\rightarrow$ solid transitions are well separated, as in [15]. The almost constant position of the onset of the liquid $\rightarrow$ solid transition indicates that neither the colour former nor the developer act as nucleation agent during the primary crystallization process of the co-solvent. On the other hand, the increased relative amounts of the colour former and developer lead to occurrence of a prolonged liquid $\rightarrow$ solid peak tail, which corresponds to formation of crystallites with larger distribution regarding their properties (size, composition, etc.). This signifies that at least one of these substances actively participates in the crystal growth process. Note that the decreasing height of the liquid $\rightarrow$ solid peak is caused by both its widening and decreasing relative amount of the cosolvent (the data are normalized with respect to the mass of the overall mixture). The successive transition between the rotator and crystal phases then exhibits significantly larger shift toward lower temperatures with increasing relative amounts of the colour former and developer and appears to be at least partially independent from the primary crystallization process. Such behaviour can be expected for the solid $\rightarrow$ solid transition kinetics, however, the exact physico-chemical mechanism responsible for this shift is unknown at the moment. If we compare the above-described data with the melting peaks obtained during the consequent heating, it can be seen that the wider distribution of the quality of the crystallites observed for lower TD concentrations very well correlates with the broadened melting exhibiting a low-temperature shoulder. Incidentally, this indicates that the rotatorto-crystal transition does not affect the overall arrangement of the crystallites formed during the preceding liquid $\rightarrow$ solid phase transformation.

Contrary to the TD data, the DSC cooling curves obtained for MA exhibit a single peak, indicating that both liquid $\rightarrow$ solid and solid $\rightarrow$ solid transitions are closely connected. In addition, the initial introduction of the colour former and developer into the MA matrix leads to accelerated formation of crystallites during cooling which then back decelerates at higher relative colour former and developer concentrations. This may indicate that these substances act as nucleating agents at very low concentrations but at the same time inhibit the system mobility due to the chemical interconnections at higher concentrations. Another possible explanation may involve a combined influences of the colour former and developer acting like nucleation agents during the liquid $\rightarrow$ solid transition and simultaneously inhibiting the consequent solid $\rightarrow$ solid transition in a similar way as in case of thermochromic composites using TD co-solvent - this second explanation would require the two transitions being kinetically dependent. Regarding the melting peaks of thermochromic composites with MA as a co-solvent, their consistent shape and relatively sharp onsets indicate that crystallites of similar shape and composition were formed during the preceding cooling step and that the cosolvent concentration has little influence on the resulting quality of the formed crystallites.

In summary, in both studied thermochromic systems, the determined onset and endset temperatures generally show slightly increasing tendency with the increasing concentration of the co-solvent, on heating as well as on cooling. For samples with TD, the overall change was about $2-4{ }^{\circ} \mathrm{C}$, whereas for samples with MA the increase was even smaller, about $1-2{ }^{\circ} \mathrm{C}$ (see Tables 1 and 2 ).

\section{Colour analysis}

All studied ternary composites exhibited thermochromic colour change, however, to different extent, as will be discussed further. The colour change of the samples with TD as a co-solvent during cooling as well as heating was gradual, in most samples with the width of hysteresis loop increasing 
towards higher temperatures, as illustrated in Fig. 4a. In the curves of $G(T)$, two major peaks can be identified, suggesting two main steps in colour change process in case of samples with lower concentrations of TD (up to $x_{\mathrm{CVL}} / x_{\mathrm{BPA}} / x_{\mathrm{TD}}=1 / 4 / 120$, complete data not shown here). The dominant one, corresponding to faster colour change, was observed at higher temperatures for both heating and cooling of these samples. The characteristic temperatures determined from gradients $G(T)$ of $\Delta E_{\mathrm{C}}$ for heating and cooling of these thermochromic composites are listed in Table 3, showing that their values varied independently on the increasing concentration of TD; nevertheless, when considering the precision and resolution of measurement available in present experimental setup for colour analysis and narrow temperature range where the concentration dependence was observed in DSC data, we cannot exclude the possibility of similar tendency in colorimetric behaviour. Decolouration of all samples was observed roughly between 20 and $40{ }^{\circ} \mathrm{C}$ with the fastest change at approx. $32{ }^{\circ} \mathrm{C}$ $\left(T_{\text {Dmax }}\right)$, while their colouration took place roughly between 38 and $19^{\circ} \mathrm{C}$ with the fastest change at approx. $30^{\circ} \mathrm{C}\left(T_{\mathrm{Cmax}}\right)$. Accordingly, the systems generally showed small width of hysteresis loop. Highest colour contrast, i.e. highest $\Delta E_{\mathrm{C}}$ between coloured and decoloured sample, approx. 170, was reached with the molar ratios of $x_{\mathrm{CVL}} / x_{\mathrm{BPA}} / x_{\mathrm{TD}}$ equal to $1 / 4 / 80$; with further increasing concentration of TD in the mixture it decreased down to the value of approx. 90 for the sample with $x_{\mathrm{CVL}} / x_{\mathrm{BPA}} / x_{\mathrm{TD}}$ $=1 / 4 / 600$.
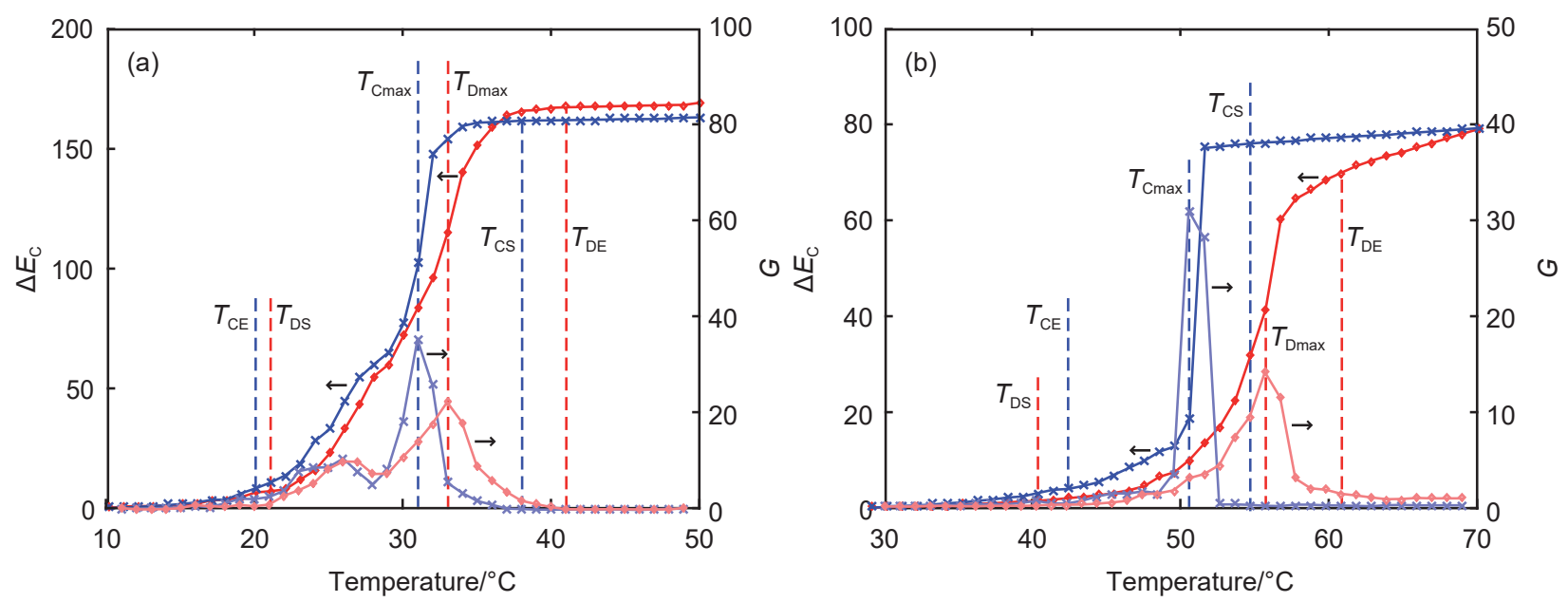

Fig. 4 Temperature dependences of cumulative colour difference $\Delta E_{\mathrm{C}}$ with corresponding gradients $G$ of the thermochromic composite using TD co-solvent with $x_{\mathrm{CVL}} / x_{\mathrm{BPA}} / x_{\mathrm{TD}}=1 / 4 / 80$ (a) and of the thermochromic composite using MA co-solvent with $x_{\mathrm{CVL}} / x_{\mathrm{BPA}} / x_{\mathrm{MA}}=1 / 4 / 200$ (b), where diamonds represent heating and crosses cooling of the sample; for the meaning of individual marked temperatures refer to Characterization in Experimental section

In thermochromic composites using MA as a co-solvent, only one major peak was observed in the curves of $G(T)$ for both heating and cooling, as demonstrated in Fig. 4b. The colour change upon cooling shows the steep drop in the whole studied concentration range; upon heating, the colour change is smoother in case of samples with higher concentrations of MA (from $x_{\mathrm{CVL}} / x_{\mathrm{BPA}} / x_{\mathrm{MA}}=$ $1 / 4 / 120$, complete data not shown here). This is reflected by wider peak in their gradients for heating run. In general, the thermochromic behaviour of samples with MA is less predictable and more time dependent than that of samples using TD co-solvent. Determined characteristic temperatures again don't show any clear dependence on the changing concentration of MA (see Table 4). Decolouration of all samples appears roughly between 44 and $60{ }^{\circ} \mathrm{C}$ with the fastest change at approx. $56{ }^{\circ} \mathrm{C}$ and their colouration takes place roughly between 55 and $42{ }^{\circ} \mathrm{C}$ with the fastest change at approx. $51{ }^{\circ} \mathrm{C}$. 
These values also indicate substantial width of hysteresis loop. Highest colour contrast, with the value around 80, was achieved with medium concentrations of MA $\left(x_{\mathrm{CVL}} / x_{\mathrm{BPA}} / x_{\mathrm{MA}}\right.$ between $1 / 4 / 100$ and 1/4/200), from which the most stable behaviour at high temperatures was observed for the molar ratios of $x_{\mathrm{CVL}} / x_{\mathrm{BPA}} / x_{\mathrm{MA}}$ equal to $1 / 4 / 200$ (see Fig. $4 \mathrm{~b}$ ). The lowest colour contrast was observed for the sample with highest concentration of MA $\left(x_{\mathrm{CVL}} / x_{\mathrm{BPA}} / x_{\mathrm{MA}}=1 / 4 / 600\right)$. Therefore, the colour contrast of thermochromic systems with MA as a co-solvent was substantially lower than that of systems using TD co-solvent in case of the whole studied concentration range.

Table 3 Colorimetric results of thermochromic composite powders with TD as a co-solvent determined characteristic temperatures for start (S), maximum rate of colour change (max) and end (E) of decolouration (D) on heating and colouration (C) on cooling

\begin{tabular}{lllllll}
\hline $\begin{array}{l}\text { Sample } \\
\left(x_{\mathrm{CVL}} / x_{\mathrm{BPA}} / x_{\mathrm{TD}}\right)\end{array}$ & $T_{\mathrm{DS}} /{ }^{\circ} \mathrm{C}$ & $T_{\mathrm{Dmax}} /{ }^{\circ} \mathrm{C}$ & $T_{\mathrm{DE}} /{ }^{\circ} \mathrm{C}$ & $T_{\mathrm{CS}} /{ }^{\circ} \mathrm{C}$ & $T_{\mathrm{Cmax}} /{ }^{\circ} \mathrm{C}$ & $T_{\mathrm{CE}} /{ }^{\circ} \mathrm{C}$ \\
\hline $1 / 4 / 60$ & 19 & 33 & 40 & 37 & 30 & 19 \\
$1 / 4 / 80$ & 21 & 33 & 41 & 38 & 31 & 20 \\
$1 / 4 / 100$ & 20 & 32 & 39 & 39 & 30 & 19 \\
$1 / 4 / 120$ & 20 & 32 & 40 & 37 & 30 & 20 \\
$1 / 4 / 200$ & 20 & 33 & 41 & 39 & 29 & 19 \\
$1 / 4 / 400$ & 20 & 33 & 41 & 40 & 31 & 20 \\
$1 / 4 / 600$ & 20 & 28 & 41 & 39 & 30 & 19 \\
\hline
\end{tabular}

Table 4 Colorimetric results of thermochromic composite powders with MA as a co-solvent determined characteristic temperatures for start (S), maximum rate of colour change (max) and end (E) of decolouration (D) on heating and colouration (C) on cooling

\begin{tabular}{lllllll}
\hline $\begin{array}{l}\text { Sample } \\
\left(x_{\mathrm{CVL}} / x_{\mathrm{BPA}} / x_{\mathrm{MA}}\right)\end{array}$ & $T_{\mathrm{DS}} /{ }^{\circ} \mathrm{C}$ & $T_{\mathrm{Dmax}} /{ }^{\circ} \mathrm{C}$ & $T_{\mathrm{DE}} /{ }^{\circ} \mathrm{C}$ & $T_{\mathrm{CS}} /{ }^{\circ} \mathrm{C}$ & $T_{\mathrm{C} \max } /{ }^{\circ} \mathrm{C}$ & $T_{\mathrm{CE}} /{ }^{\circ} \mathrm{C}$ \\
\hline $1 / 4 / 60$ & 46 & 55 & 57 & 52 & 51 & 40 \\
$1 / 4 / 80$ & 40 & 55 & 60 & 53 & 52 & 41 \\
$1 / 4 / 100$ & 45 & 55 & 59 & 59 & 50 & 41 \\
$1 / 4 / 120$ & 50 & 55 & 58 & 61 & 50 & 46 \\
$1 / 4 / 200$ & 41 & 56 & 61 & 55 & 51 & 43 \\
$1 / 4 / 400$ & 39 & 57 & 61 & 54 & 51 & 39 \\
$1 / 4 / 600$ & 47 & 56 & 61 & 54 & 52 & 43 \\
\hline
\end{tabular}

\section{Combined assessment}

The two methods employed for evaluation of dynamic behaviour of investigated thermochromic systems complement each other, as illustrated in Fig. 5. In case of thermochromic systems using MA co-solvent, the temperature, which corresponds to the largest decolouration, lies very close to the endset of the solid $\rightarrow$ liquid transition $T_{\mathrm{EH} 1}$. Similarly, the temperature corresponding to the point with the largest colouration gradient lies near the endset of liquid $\rightarrow$ solid transition. Samples containing smaller amount of MA were still coloured after the thermochromic system melted and no stable 
decolouration limit was obtained. However, the most apparent colour change can be directly connected to the phase change of MA co-solvent.
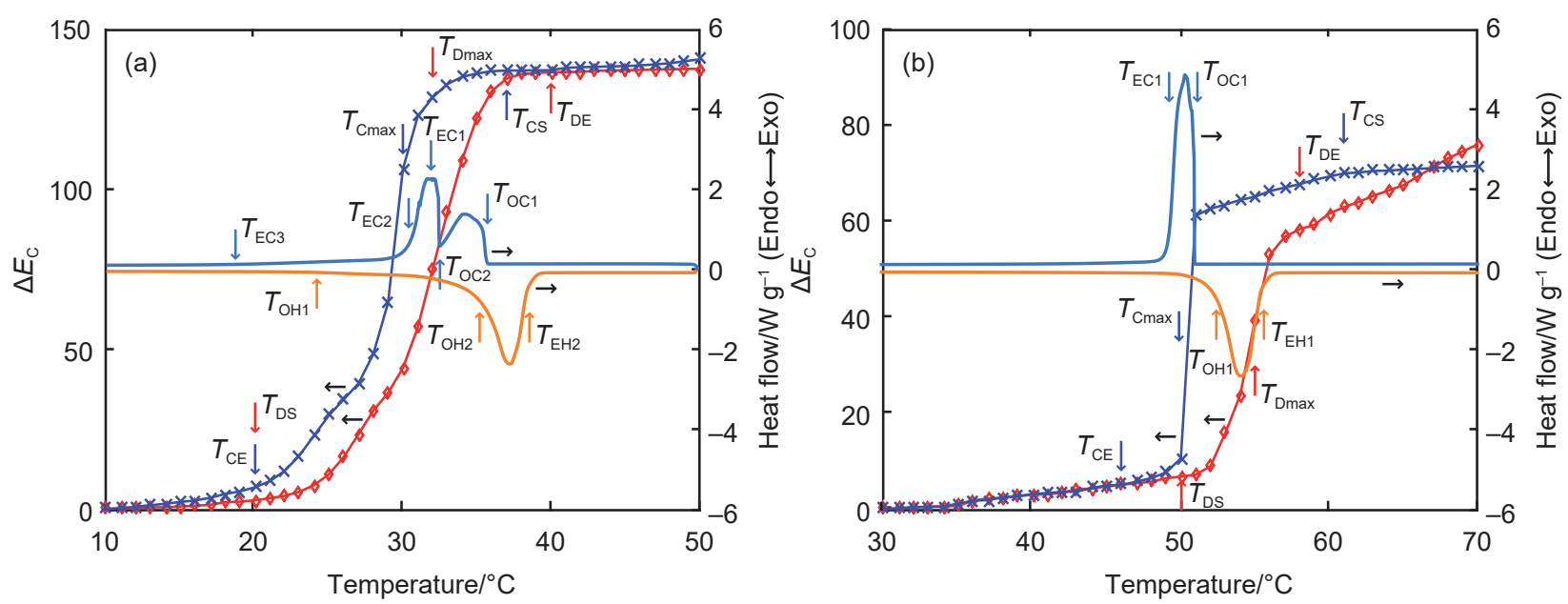

Fig. 5 Combined colour and thermal analyses of the thermochromic composite using TD co-solvent with $x_{\mathrm{CVL}} / x_{\mathrm{BPA}} / x_{\mathrm{TD}}=1 / 4 / 120$ (a) and of the thermochromic composite using MA co-solvent with $x_{\mathrm{CVL}} / x_{\mathrm{BPA}} / x_{\mathrm{MA}}=1 / 4 / 120(\mathrm{~b})$, where diamonds and endothermic curve represent heating and crosses and exothermic curve represent cooling of the sample in cumulative colour difference and DSC representation, respectively; for the meaning of individual marked temperatures refer to Characterization in Experimental section

Systems containing TD as a co-solvent became decoloured always after complete liquefaction even if large amount of the co-solvent was present in the system. While the liquid $\rightarrow$ solid phase transition is connected with only slight colouration, the substantial colour change corresponds to the solid $\rightarrow$ solid transition. Because the liquid $\rightarrow$ solid and solid $\rightarrow$ solid phase transitions are more separated on cooling, the gradient of dynamic colour change exhibits sharper shape. The flat shoulder occurring in DSC curves of samples with the increased relative amounts of the colour former and developer at temperatures below the solid $\rightarrow$ solid transition peak has its counterpart in corresponding curves of cumulative colour difference $\Delta E_{\mathrm{C}}$. At present, more thorough interpretation was not possible due to the lack of precision in colorimetric measurement of dynamic colour change when compared to information available from DSC measurement. This issue is also discussed in [19], where the investigation of thermochromic systems comprising CVL, BPA, and TD is extended by IR analysis, including corresponding binary systems.

\section{Conclusions}

Two sets of thermochromic systems, containing long-chain aliphatic alcohol and acid as a co-solvent, were prepared and analysed in terms of dynamic colour change and phase transitions. In case of alcoholic co-solvent, the temperature at which the colour change stops on heating $\left(T_{\mathrm{DE}}\right)$ is closely connected with the temperature of the solid $\rightarrow$ liquid transition of the thermochromic composites. Similarly, the temperature at which the colouration starts $\left(T_{\mathrm{CS}}\right)$ is connected with the temperature of the liquid-solid transition upon cooling. The most rapid colour change $\left(T_{\mathrm{Cmax}}\right)$ lies near the solid $\rightarrow$ solid phase transition of the alcoholic co-solvent. Temperatures corresponding to the lower limit of the colour change range, i.e. the temperature at which the decolouration starts on heating $\left(T_{\mathrm{DS}}\right)$ and the temperature at which the colour change stops on cooling $\left(T_{\mathrm{CE}}\right)$, cannot be directly 
related to the phase transition temperatures of thermochromic systems obtained by DSC, but are reflected in the flat shoulder in corresponding temperature range. The temperatures characterizing the rapid colouration and decolouration of thermochromic systems using MA co-solvent are correlated with the endset of the liquid $\rightarrow$ solid and solid $\rightarrow$ liquid transition, respectively. The connection between thermal and colour analysis is slightly different for alcoholic and acidic co-solvent. Further analysis and evaluation of temperature ranges of individual phase transitions necessary to understand the mechanism of colour change both on heating and cooling would require a substantial change of experimental setup enabling measurement of dynamic colour change with higher temperature resolution and with more heating and cooling rates.

\section{References}

[1] Panák O, Hauptman N, Klanjšek Gunde M, Kaplanová M. Colorimetric characterisation of thermochromic composites with different molar ratios of components. J Print Media Technol Res. 2012;1:113-20.

[2] Panák O, Držková M, Kaplanová M. Insight into the evaluation of colour changes of leuco dye based thermochromic systems as a function of temperature. Dyes Pigm. 2015;120:279-87.

[3] Hajzeri M, Bašnec K, Bele M, Klanjšek Gunde M. Influence of developer on structural, optical and thermal properties of a benzofluoran-based thermochromic composite. Dyes Pigm. 2015;113:754-62.

[4] Tang H, MacLaren DC, White MA. New insights concerning the mechanism of reversible thermochromic mixtures. Can J Chem. 2010;88:1063-70.

[5] Bourque AN, White MA. Control of thermochromic behaviour in crystal violet lactone (CVL)/alkyl gallate/alcohol ternary mixtures. Canad J Chem. 2015;93:22-31.

[6] Burkinshaw SM, Griffiths J, Towns AD. Reversibly thermochromic systems based on pH-sensitive functional dyes. J Mater Chem. 1998;8:2677-83.

[7] Bašnec K, Hajzeri M., Klanjšek Gunde M. Thermal and colour properties of leuco dye-based thermochromic composite with dodecanol solvent. J Therm Anal Cal. 2016; doi 10.1007/s10973-016-5670-9

[8] Tasumi M, Shimanouchi T, Watanabe A, Goto R. Infrared spectra of normal higher alcohols-I. Spectrochim Acta. 1964;20:629-66.

[9] Sirota EB, Wu XZ. The rotator phases of neat and hydrated 1-alcohols. J Chem Phys. 1996;105:7763-73.

[10] Ventolà L, Ramírez M, Calvet T, Solans X, Cuevas-Diarte MA. Polymorphism of n-alkanols: 1-Heptadecanol, 1octadecanol, 1-nonadecanol, and 1-eicosanol. Chem Mater. 2002;14:508-17.

[11] Ventolà L, Calvet T, Cuevas-Diarte MA, Solans X, Mondieig D, Négrier P, van Miltenburg JC. Solid state equilibrium in the n-alkanols family: the stability of binary mixed samples. Phys Chem Chem Phys. 2003;5:947-52.

[12] Ventolà L, Calvet T, Cuevas-Diarte MA, Oonk HAJ, Mondieig D. Solid-solid and solid-liquid equilibria in the nalkanols family: $\mathrm{C}_{18} \mathrm{H}_{37} \mathrm{OH}-\mathrm{C}_{20} \mathrm{H}_{41} \mathrm{OH}$ system. Phys Chem Chem Phys. 2004;6:3726-31.

[13] Sirota EB, King HE Jr, Shao HH, Singer DM. Rotator phases in mixtures of n-alkanes. J Phys Chem. 1995;99:798804.

[14] van Miltenburg JC, Oonk HAJ. Heat capacities and derived thermodynamic functions of 1-octadecanol, 1nonadecanol, 1-eicosanol, and 1-docosanol between $10 \mathrm{~K}$ and $370 \mathrm{~K}$. J Chem Eng Data. 2001;46:90-97.

[15] Zeng JL, Cao Z, Yang DW, Xu F, Sun LX, Zhang L, Zhang XF. Phase diagram of palmitic acid-tetradecanol mixtures obtained by DSC experiments. J Therm Anal Cal. 2009;95:501-505.

[16] Sarı A. Thermal reliability test of some fatty acids as PCMs used for solar thermal latent heat storage applications. Energy Convers Manag. 2003;44:2277-87.

[17] Gandolfo FG, Bot A, Flöter E. Phase diagram of mixtures of stearic acid and stearyl alcohol. Thermochimica Acta. 2003;404:9-17.

[18] Rochester JR. Bisphenol A and human health: A review of the literature. Reprod Toxicol. 2013;42:132-155.

[19] Panák O, Držková M, Kaplanová M, Novak U, Klanjšek Gunde M. The relation between colour and structural changes in thermochromic systems comprising crystal violet lactone, bisphenol A, and tetradecanol. Dyes Pigm. 2016; doi 10.1016/j.dyepig.2016.08.050 